\title{
Las relaciones internacionales del Perú a través del diario Perú21. Encuadres noticiosos en la información periodística sobre asuntos de Cancillería
}

Recibido: 04 de agosto de 2015

Aceptado: 18 de marzo de 2016

Publicado: 30 de noviembre de 2016
Carlos Ricardo Gonzales García

carlos.gonzales1@unmsm.edu.pe

Universidad Nacional Mayor de San Marcos (Perú)

Resumen: Este artículo tiene como objetivo analizar los contenidos periodísticos del diario Perú21, tomando como foco de interés las informaciones sobre las relaciones internacionales para conocer los frames o encuadres episódicos y temáticos presentes en las noticias, así como la aproximación que guardan con la agenda oficial de asuntos exteriores. El corpus consistió en 1295 textos periodísticos producidos entre los meses de julio de 2011 y julio de 2015. Los diferentes encuadres se determinaron sobre la base de la teoría del framing y la tipología de frames propuesta por Semetko y Valkenburg. Los resultados indican que la producción noticiosa de los asuntos de Cancillería se define fundamentalmente por la "atribución de responsabilidad" y la "consecuencia económica", particularmente en temas de libre comercio y economía de mercado, seguridad y defensa; derechos humanos, combate contra el narcotráfico, medio ambiente y cooperación técnica.

Palabras clave: Relaciones exteriores, Cancillería, encuadres periodísticos, periodismo peruano, política, economía.

Abstract: This article aims to analyze the journalistic content of the newspaper Perú21, on the focus of interest information on international relations to know the frames or episodic and thematic frames in the news, and the approach saved with the official agenda offoreign affairs. The corpus consisted of 1295 journalistic texts produced between the months of July 2011 to July 2015. The different framings were determined on the basis of the theory of framing and typology of frames proposed by Semetko and Valkenburg. The results indicate that the news production issues Chancellery is defined primarily by the "attribution of 
responsibility" and "economic consequences", particularly on issues of free trade and market economy, security and defense, human rights, fight against drug trafficking, the environment and technical cooperation.

Key words: Foreign Affairs, Foreign Ministry, Journalistic Framing, Peruvian Journalism, Politics, Economy.

\section{Introducción}

Al considerarse la relación existente entre élite política y medios de comunicación en la esfera de la política exterior (García Marín, 2007) se identifican cuatro modelos. En el primero de estos ("modelo de propaganda") los medios de comunicación se convierten, fundamentalmente, en portavoces de los intereses de las élites políticas y económicas de la sociedad. Otro, es el denominado "efecto $C N N$ ", que establece que la cobertura mediática de un asunto, generalmente conflictivo, puede obligar a los diferentes gobiernos a actuar independientemente de la agenda que tuvieran planeada. El tercero es el llamado "modelo indexado" que, partiendo de las bases del modelo de propaganda, refiere que los medios de comunicación refleja el alcance del debate político, situando la variable independiente en el consenso político y la dependiente en la cobertura mediática. Finalmente, existe un cuarto modelo que lleva por nombre "cascada", donde se rompe el monopolio de las relaciones entre las élites y los medios de comunicación como marco explicativo fundamental para conocer el proceso de comunicación política, dando cabida a otros actores como la opinión pública, aunque situada al nivel más bajo de su influencia.

Esta investigación, sobre la propuesta metodológica de la teoría del framing o encuadres noticiosos, establece como modelo de comunicación para el estudio de la cobertura de las relaciones internacionales del Perú con el mundo, todos estos marcos explicativos, a fin de conocer las relaciones existentes entre los "temas de la noticia" presente en la producción mediática y sus frames, entendidos estos como elementos de análisis que permiten realizar abordajes deductivos, a través de un listado de ítems preestablecido.

De esta manera, este trabajo pretende analizar fundamentalmente el contenido de los mensajes de las informaciones ubicadas en las distintas secciones de un diario de circulación nacional para identificar (mediante la metodología de encuadres periodísticos de Semetko y Valkenburg) los enfoques o frames más utilizados en la representación de la realidad de aquellos países con los cuales tenemos vínculos políticos, económicos, culturales a través de límites, acuerdos, tratados, negociaciones, entre otros.

\section{Objetivos}

- Establecer los frames y la estructura con que son presentados los temas de relaciones internacionales en Perúz 1 .

- Identificar qué regiones y países, según el medio elegido, son los que mayormente 
tienen mayor vínculo político, económico, social y cultural con el Perú de acuerdo con la agenda de relaciones exteriores de Cancillería.

- Describir la frecuencia y el espacio destinado a las informaciones sobre relaciones exteriores, en cuanto a temas, encuadres, promotores, situación de las fuentes, países y regiones.

\section{Metodología}

\subsection{Diseño metodológico}

El trabajo de investigación es observacional, de diseño no experimental, dado que no es posible la manipulación de ninguna de sus variables. El enfoque es de tipo cuantitativo, por el tamaño y la selección de la muestra. A partir de la revisión de las informaciones publicadas en los cuatro últimos años por un diario de circulación nacional, se cuantificó los puntajes obtenidos para realizar el contraste de la hipótesis planteada.

Es así como se efectuó un diseño de tipo descriptivo basado en el análisis de contenido sobre las relaciones internacionales del Perú con el resto del mundo que nos permitió estudiar (desde un aspecto cualitativo, a nivel deductivo e inductivo) los discursos y encuadres en la prensa escrita sobre el conjunto de interacciones políticas, económicas, sociales y culturales del país con el resto de la sociedad internacional.

\subsection{Universo y muestra}

Para el presente estudio, el universo estuvo integrado por 1460 ediciones del diario Perú21 publicados entre los meses de julio de 2011 y julio de 2015. Con respecto a la muestra, se seleccionaron las informaciones publicadas por Perú 21 referidas a las relaciones internacionales del Perú con el resto del mundo. De esta manera, se comenzó a contar desde el 28 de julio (inicio del gobierno de Ollanta Humala) hasta el 28 de julio de 2015, en que se cumplieron cuatros años de su gestión presidencial.

El proceso de búsqueda dio como resultado la detección de 1295 notas o unidades de análisis sobre las relaciones internacionales del Perú con el resto del mundo (países, continentes, organizaciones, entre otras). La muestra está conformada, sin importar el espacio que ocupen (con o sin fotografía), por notas vinculadas a las relaciones internacionales entre el Perú y un país u organización según la agenda exterior del Estado peruano y cuyos formatos sean noticias, entrevistas, comentarios, crónicas o reportajes aparecidos en las secciones "Portada", "Actualidad", "Política", "Economía", "Mundo", "Ciudad" y "Perú".

\subsection{Técnicas de recolección de datos}

La construcción de un manual de codificación nos permitió elaborar una ficha de análisis compuesta por 5 apartados básicos: 
- Datos de identificación básicos, tales como identificación de la unidad de análisis, fecha de publicación (AA/MM/DD).

- Caracterización de las informaciones, que toma en cuenta una adaptación de los puntos focales propuesto por Tankard (2001) para medir los encuadres noticiosos (tipo de unidad, lugar en la página, espacio y contextualización gráfica).

- Política exterior, que implica la identificación del continente y país, así como del tema vinculado a la agenda de la Cancillería.

- Promotores y situación de la fuente.

- Encuadre general según la metodología de Semekto y Valkenburg (atribución de responsabilidad, conflicto, interés humano, moralidad y consecuencias económicas).

\subsection{Técnicas para el procesamiento y análisis de la información}

Para la organización, procesamiento y presentación de datos se usó el programa MS Excel (hoja de cálculo) y el software estadístico SPSS. La primera cuantificación se hizo sobre la base de un nivel nominal, evaluándose la frecuencia de adscripción de las unidades de análisis con la tipología de encuadres de Semetko y Valkenburg.

La segunda cuantificación se hizo a dos niveles: a) de razón, sujeto a factores de prioridad y esquema propio, midiéndose espacios por líneas, párrafos y considerándose la ubicación (por sección) de la nota en el diario y la presencia de recursos gráficos; y b) nominal, evaluándose la frecuencia de adscripción o coincidencia de las unidades de análisis con encuadres inductivos sean episódicos o temáticos.

En ambas cuantificaciones, se evaluó el grado de proximidad con la agenda de relaciones exteriores del Estado peruano. Cabe destacar que la codificación de las unidades de ambas hipótesis se hizo mediante la utilización de una plantilla por cada nota periodística.

\section{Hipótesis de trabajo}

La selección y prominencia de determinados temas, actores y atributos (así como el encuadre utilizado para su presentación) prioriza, en cuanto a las relaciones internacionales del Perú, la responsabilidad de funcionarios y organismos oficiales en asuntos políticos globales, conflictos en asuntos de soberanía y límites, así como en consecuencias en asuntos económicos. Por tanto, si la opinión pública nacional dota de realidad al relato, construido sobre la base de estos encuadres, las consecuencias a nivel cognitivo estarán en razón del poder y del lucro del comercio global.

\section{La teoría del encuadre (Framing Theory)}

El análisis del tratamiento informativo de las relaciones internacionales del Perú ha sido abordado desde la framing theory ("teoría del encuadre") en dos tesis defendidas por 
el autor de este artículo en la USMP y en la UNMSM (Gonzales, 2015 y 2016). Desde estos estudios, se conciben los géneros periodísticos (principalmente la noticia) como la representación de hechos que dependen de un determinado frame ("enfoque") con el que se otorga ciertos rasgos, atributos o características sobre un tema (Pinto, 2009), dando más énfasis a unas posturas frente a otras (Prieto, 2013) y que no son más que el resultado de una definición de problemas (Entman, 1993) a fin de hacerlo más comprensible para el público, pudiendo generar efectos de tipo cognitivo y afectivo sobre las actitudes del público que los consume (Muñiz, 2015).

En este "proceso de semantización" (Verón y otros, 1971) no todo lo que seleccionan y valoran los profesionales de la información son "hechos", entendidos como "secuencias unitarias del acontecer" (Armentia y Caminos, 1998) sino que, de acuerdo con Gomis (2008), se tratan de "pseudoeventos" o hechos provocados que, a menudo, se inscriben en un marco ya previsto para él, es decir, que se producen con la intención primera que sean noticia, sin otro fin que llamar la atención. En el análisis de las informaciones publicadas abunda este tipo de textos, por ejemplo, en conferencias de prensa, giras diplomáticas, acuerdos o reuniones.

El news frame ("encuadre noticioso") es la demostración de que los profesionales de la información (a través de sus propios medios) se erigen como constructores de la realidad o, como lo entiende Rodrigo Alsina (2005), "productores de conocimientos", lo cual permite definir cómo y qué sabrán las personas al seleccionar e interpretar los hechos. Esto lleva a considerar los medios de comunicación y el proceso de framing como "un proceso social con especial incidencia sobre la opinión pública" (Rodríguez Pérez, 2015: 70).

\section{La noción de framing}

Pese a que existen numerosos estudios sobre los encuadres noticiosos (DeVreese, 2005), es difícil encontrar una definición consensuada sobre el significado del término "encuadre" (Aruguete y Zunino, 2010). Para empezar, existe una variedad de acepciones que recibe dicha noción, tales como "marco", "formato", "cuadro", "enfoque" (Sádaba, 2001 y Amadeo, 2008). No obstante, ambos autores reconocen que preferirían el empleo de la denominación "encuadre" como equivalente a frame, pues entienden que ambos términos responden al mismo objeto de análisis, sobre todo cuando se refieren a los medios de comunicación.

Asimismo, otro problema que no favorece a su definición es la postura de algunos autores, como Etman (1993), quienes señalan que el análisis de los encuadres noticiosos se debería desligar de la teoría de la agenda-setting (propuesta por McCombs) a fin que se convierta en un área de investigación con entidad propia. Matthes y Kohring piensan que este debate teórico, que se dio intensamente hasta fines de la década de los noventa, se encuentra ahora en pleno periodo de consolidación: 
"En este comienzo de siglo se observan intentos solventes de reorganización teórica de toda la especialidad. Además, esta iniciativa clarificadora se realiza en un contexto de expansión empírica todavía más intenso que en la década anterior, como se ha señalado previamente. Respecto a la reorganización, este periodo viene marcado por la aparición de importantes obras de referencia (Scheufele, 1999, 2000; Reese, Gandy y Grant, 2001; D’Angelo, 2002), textos que abordan la revisión teórica de todo lo realizado hasta el momento desde una perspectiva global y con dos objetivos claros: determinar los principales problemas que han impedido la consolidación hasta el momento y afrontar los retos de clarificación conceptual y metodológica que plantean. Los últimos hitos de esta reorganización los encontramos en el monográfico de Journal of Communication ya citado (Tewksbury y Scheufele, 2007) y en un reciente estudio panorámico sobre la cuestión metodológica del framing" (ápud Vicente y López, 2009: 19).

Para ganar en precisión conceptual, se va a definir el término "encuadre" como marco aplicado a la agenda de los medios. Como lo ha planteado Iguartua y otros (2005: 6), es el

“ángulo, enfoque, perspectiva o tratamiento de una información que se manifiesta en la elección, énfasis o importancia atribuida a los diferentes elementos que conforman un texto comunicativo (entre ellos, los lugares geográficos nombrados, espacios físicos y temporales mencionados, actores protagonistas descritos, definiciones de los actores y las acciones realizadas) y la forma cómo cobrarían dichos elementos más o menos enfatizados en un texto".

De este modo, la teoría del framing explica que los medios de comunicación son decisivos para interpretar la realidad (Rodríguez Pérez, 2014). Además de seleccionar los temas de la agenda periodística y jerarquizar sus noticias, se preocupan por decidir cuál será el foco principal de sus textos. Dicho trabajo de producción periodística implica que en los hechos se utilice un ángulo determinado o una perspectiva concreta conocida como marco, encuadre o enfoque.

A partir de lo dicho, según Tankard (2001), el encuadre noticioso es la idea organizativa central del contenido de las noticias que proporciona un contexto y sugiere cuál es el significado dado a los temas, mediante el uso de la selección, el énfasis, la exclusión y la elaboración de ciertos aspectos o elementos que están interrelacionados y organizan el discurso de las historias. Todorov señala que el narrador elige una forma de presentar la historia, es decir, la "realidad" evocada por el texto. Por ejemplo, decide cómo organizar los hechos (qué contar primero y qué contar después), dónde detenerse más y qué elementos excluir. Es lo que él llama discurso. "Y esa forma, el discurso, produce sentidos tanto como lo produce el 'contenido', la historia, solo que su funcionamiento no es legible a simple vista” (ápud Peralta y Urtasun, 2007: 191-192).

Para Gamson y Modigliani (1989: 3), el frame es "la idea central organizadora que da sentido a los acontecimientos y sugiere cuál es la cuestión tratada". Por su parte, Reese 
define con el término "encuadre" como todos aquellos principios organizadores socialmente compartidos y constantes en el tiempo, que trabajan simbólicamente para constituir el mundo social de modo significativo (ápud Aruguete y Zunino, 2010).

Frente a estas posturas, Entman (1993), quien ha dado mayor sustento a la teoría del framing, según Muñiz (2015), señala que los distintos encuadres que son enfatizados en las noticias proporcionan una descripción de los asuntos o problemas, diagnostican causas, realizan juicios morales y sugieren soluciones o remedios. En tal sentido, "encuadrar" es seleccionar algunos aspectos de una realidad percibida y volverlos más resaltantes en el texto que se comunica, de manera que, al determinar su agente causal (D'Adamo y García Beaudoux, 2009), promueva una definición del problema determinado junto con su interpretación casual, su evaluación moral o la recomendación de cómo debe ser tratado para el asunto descrito.

La selección y la relevancia, en conclusión, son los dos elementos primordiales que configuran la teoría del framing. En un estudio reciente, Hanggli y Kriesi (2012) plantean que el encuadre es el conjunto de argumentos, palabras o imágenes que el profesional de la información usa cuando elabora información que proviene de sus fuentes (Muñiz, 2015). De esta forma, los frames, cuando destacan posiciones sobre un tema, permiten establecer interpretaciones sobre asuntos que se encuentran en los mismos textos de las noticias.

\section{La comunicación política internacional}

Según Ochoa (2000: 147), las relaciones internacionales entre los países se ven actualmente influidas no solo por aspectos de la política económica y social (y por las acciones que sus miembros emprenden a través de ONG o incluso grupos de terrorismo), sino también por la imagen que los medios de comunicación presentan.

Dentro de ese enorme ámbito de relaciones sociales, la comunicación política busca el bien común para todas las naciones, las iniciativas de paz y la planeación de proyectos de apoyo y desarrollo económico e infraestructura. Sin embargo, como se verá más adelante en el capítulo correspondiente a los frames de los medios periodísticos, estos objetivos privilegiados dentro del terreno de lo diplomático ocupan, en el mejor de los casos, espacios secundarios en toda la subregión y casi nulos en el resto del continente, por lo que requieren de una estrategia especial.

Lograr el consenso o la adhesión de un mayor número de países a las iniciativas generadas por la política interna de un país conduce a que estas se reconozcan o acepten como normas, principios y formas de convivencia universales. Para ello, la información sobre decisiones y acciones de un país es fundamental en todo este proceso pues es así como se puede intervenir, mediar, negociar o proponer alternativas de solución a los países que se encuentren en un conflicto o se hallen inmersos en una negociación. La comunicación internacional es, como lo define Sahagún, como 
“el proceso relacional en virtud del cual dos o más actores internacionales, partiendo de experiencias históricas compartidas y de bases culturales comunes, son capaces de intercambiar información, influyéndose recíprocamente y contribuyendo a modificar la estructura dinámica de la sociedad internacional en que se encuentren inmersos" (ápud Ochoa, 2000: 148).

\subsection{Las culturas en el contexto de la comunicación política}

En el orden internacional, las culturas, en tanto subsistemas artificiales creados y concretos de toda sociedad humana (Bunge, 2007), se pueden ver afectados o influenciados por otros más hegemónicos. Esta situación hace poco probable la difusión de culturas pertenecientes a países subdesarrollados, pues al ser ocupados los espacios donde se desarrollan, tienden a desaparecer del territorio social.

Según Ochoa (2000), las iniciativas políticas para preservar las aportaciones culturales son ampliamente valoradas en el ámbito internacional. No obstante, eso no le exime a las culturas de sociedades menos avanzadas a integrarse en el marco de otras más amplias y complejas como sucede actualmente con la parte comercial, a través de tratados de libre comercio u otros tipos de acuerdos comerciales. Para ello, no cabe duda, que se requieran mecanismos políticos que faciliten la convivencia, el respeto mutuo y la participación, de tal forma que no se generen conflictos étnicos o territoriales.

Con frecuencia ocurre que las disputas entre dos o más países tienen raíces históricas, tal como sucede en el contexto sudamericano entre Chile y el Perú, y por ello se considere que las diferencias sean insalvables. Ante tales amenazas, la mediación de organismos legitimados ante la opinión pública internacional puede solucionar o aminorar en algún grado tales diferencias. Aun cuando el término de "organismo internacional" con frecuencia se identifica con la ONU, este se refiere a un fenómeno de mayor importancia, pues si define en su sentido más amplio (como cualquier grupo de individuos provenientes de al menos dos países y que disponen de un aparato institucional formal que facilita la interacción ordinaria entre los miembros a través de las fronteras nacionales) existen literalmente miles de tales entidades en el mundo. Pearson y Rochester (2000) nos dicen que los organismos internacionales se pueden clasificar de acuerdo con tres criterios básicos: a) miembros o composición, b) alcance geográfico y c) alcance funcional.

\subsection{La globalización y sus efectos en la comunicación política}

Se entiende a la globalización como el proceso de integración, primero económica y comercial, después cultural y política entre países de todo el mundo. El concepto implica considerar una serie de exigencias de carácter comunicativo, de manejo de la información, de formación de individuos, de organización de actividades comerciales, entre otras que permiten un mayor acercamiento y por tanto una mejor integración de todo tipo de recursos entre los países. 
El tema, sin duda, ha sido abordado con amplitud dentro de diversos campos disciplinarios como el de la sociología, la ciencia política, la economía, las relaciones internacionales y la industria de la información y la cultura. Sánchez-Ancochea (2007) amplía esta cuestión por el lado de los efectos de la globalización, tanto en países desarrollo como en países en desarrollo. Desde una perspectiva hermenéutica y crítica, Urreiztieta (2004) analiza los procesos psicosociales más relevantes relacionados con la configuración de nuevos proyectos de subjetividad y políticas de vida.

Esto ha llevado a que se vuelva "casi ortodoxo" el referirse a la crisis de gobernabilidad de los Estado-nación, como afirma Smelser (2003), situación impuesta por la lógica de la economía global y la proliferación de una nueva lógica en la toma de decisiones que involucra a organizaciones intergubernamentales e internacionales, tales como la Unión Europea, el Fondo Monetario Internacional, organizaciones internacionales ambientales y otras de tipo no gubernamental.

No hay que olvidar el papel que juegan las comunicaciones quienes han logrado un desarrollo global. El desarrollo de la tecnología en dichos medios ha hecho posible llegar (cada vez más lejos y más rápido) a mayor número de gente. Los cambios se presentan con una aceleración vertiginosa por medio del desarrollo de redes globales de comunicación estructuradas e información de alcance global. Asimismo, otros servicios de telecomunicaciones han permitido aumentar las posibilidades de comunicación internacional, de manera directa, entre ciudadanos de distintos países.

Ochoa (2000: 152) afirma que los acontecimientos y las declaraciones de los políticos son presentados en orden jerárquico en un esquema que configura el orden del mundo: "así podemos entender que la información local ocupe ciertos espacios. Pero la información nacional implica otro nivel de relevancia, y la internacional tiene también su propio lugar". Sin embargo, eso no significa que puedan tener la misma trascendencia. Por ejemplo, las declaraciones del presidente de los Estados Unidos serán más importantes que las del mandatario de Filipinas o de cualquier país del África. Los medios de comunicación clasifican y organizan la información mundial basándose en jerarquías, relevancias o trascendencia y novedades.

\section{Resultados}

- Cobertura: tomando como referencia la lista de países que maneja el Ministerio de Relaciones Exteriores, hay un buen porcentaje de países ausentes en la cobertura periodística de Perú21. Son 57 naciones que representan la mitad del total (50\%) y que se encuentran en América Central y el Caribe ${ }^{1}$, en la Europa Comunitaria ${ }^{2}$, en la Europa

1 Nicaragua, Barbados, Jamaica, Las Bahamas, y Trinidad y Tobago.

2 Austria, Bulgaria, Chipre, Dinamarca, Eslovaquia, Eslovenia, Estonia, Finlandia, Grecia, Hungría, Irlanda, Letonia, Lituania, Luxemburgo, Malta y Polonia. 
no Comunitaria ${ }^{3}$, en Asia y Oceanía ${ }^{4}$ y en África, Medio Oriente y Golfo ${ }^{5}$. En cuanto al número de unidades, Chile y Bolivia son los únicos países que superan los cien textos ${ }^{6}$. Estados Unidos se ubica tercero en la lista, que posee 94 unidades (7\%).

- Uso de géneros periodísticos: el género más utilizado es el de la noticia(1203 unidades), que representan el 93\% del total abarcado. Le siguen las columnas de opinión (4\%) y los reportajes $(2 \%)$.

- Secciones que ocupan los hechos: "Política" es la sección donde aparecen con mayor frecuencia los textos sobre relaciones internacionales $(62 \%)$. Su relevancia crece cuando 47 unidades (15\%) de 319 textos contaron con "Portada". Otra sección que cuenta con visibilidad es "Economía" (25\%). Ambas hacen un total de 1122 unidades, lo que representa el $87 \%$.

- Espacio por región y país: el 79\% de las notas periodísticas ocuparon un espacio de menos de media página. Del total, las notas breves representan el 50\%.

- Uso de recursos gráficos: la fotografía es el recurso que utiliza más Perú21 para contextualizar la información que publica. Sin embargo, el porcentaje de textos que carecen de este $u$ otro recurso gráfico es muy alto (48\%).

- Fuentes informativas: la principal promotora de las relaciones internacionales es la Cancillería (13\%), seguida muy de cerca por Presidencia de la República (12\%). Otros actores importantes son los otros miembros del Ejecutivo (8\%), empresas y gremios (7\%), como la Asociación de Exportadores y la Cámara de Comercio de Lima.

- Situación de la fuente: un porcentaje importante (85\%) pertenece a las actividades del reporterismo (o en tareas de corresponsalía o agencia). Los comunicados de prensa y documentos oficiales ocupan una posición muy pequeña en el trabajo con los promotores de la información (4\%).

- Textos por tema principal: en los 1295 textos, se han detectado 523 unidades que corresponden, según la Agenda de Política Exterior de Cancillería, a todos los temas que abarcan "asuntos políticos" (40\%), "asuntos económicos" (32\%), "soberanía, límites y asuntos antárticos" (16\%) y “asuntos sociales”, con solo 5\%.

3 Albania, Andorra, Azerbaiyán, Bosnia-Herzegovina, Croacia, Georgia, Kosovo, Liechtenstein, Macedonia, Moldavia, Mónaco, Montenegro, San Marino, Serbia y Soberana Orden Militar de Malta.

4 Australia, Filipinas, República de la Unión de Myanmar, Mongolia, Nepal, Sri Lanka, Bangladesh, Laos, Timor Oriental, Brunei Darussalam, Maldivas, Camboya, Papúa Nueva Guinea, República de Fiji y República de Vanuatu.

5 Túnez, Libia, Marruecos, Mozambique, Ghana y Sudán.

6 Chile tiene 278 (22\%) y Bolivia $105(8 \%)$. 
- Frames utilizados por tema en la cobertura periodística: los asuntos políticos son mayormente encuadrados en el frame de "atribución de responsabilidad" (86\%); los asuntos económicos en los encuadres de "consecuencias económicas" (56\%) y "atribución" (40\%); los asuntos de soberanía, límites y asuntos antárticos en los enfoques de "atribución" (68\%) y "conflicto" (27\%); los asuntos sociales en los frames de "atribución" (88\%); los temas de comunidades peruanas en el exterior en los encuadres de "atribución" (69\%) e "interés humano" (27\%) y los Asuntos culturales y tratados / acuerdos / declaraciones en los enfoques de "atribución” (78\% y 75\% respectivamente).

\section{Conclusiones}

Se pudo comprobar (en su mayor parte) la hipótesis de la investigación, ya que las noticias que produce el diario Perú.21 (principalmente en las secciones "Política" y "Economía") lo hacen efectivamente con los encuadres de "atribución de responsabilidad" y "consecuencias económicas". Estos encuadres deductivos genéricos presentes en las informaciones (de tipo temático) guardan correspondencia con la agenda oficial del Estado peruano en asuntos exteriores, específicamente, en temas como libre comercio y economía de mercado, seguridad y defensa, derechos humanos combate contra el narcotráfico, medio ambiente y cooperación técnica.

Con relación a los temas presentes en las pautas de nuestro medio (tanto por continente y país, así como por organización internacional) podemos concluir que existe una alta tendencia a tratar sobre las mismas temáticas (asuntos políticos y económicos) como si estas fueran la únicas dimensiones, descuidándose aspectos como asuntos culturales (política cultural, promoción cultural, relaciones educativas y del deporte), comunidades peruanas en el exterior y asuntos sociales (desarrollo social, niñez, adolescencia y juventud, mujer, adulto mayor, pueblos indígenas y afrodescendientes, salud, discapacidad, empleo y trabajo, seguridad alimentaria, Objetivos de Desarrollo del Milenio y de Desarrollo Sostenible y Agenda Post 2015).

De acuerdo con las unidades de análisis recogidas, América Latina (principalmente Sudamérica) se diferencia, en número de notas y espacio, por un amplio margen del resto del mundo, siendo sobre todo los países fronterizos (especialmente Chile) y Bolivia, los más privilegiados por la agenda política y mediática peruana, colocándose más atrás los Estados Unidos. En el caso de los países de la Europa comunitaria y no comunitaria, tenemos a España y Rusia, respectivamente. En cuanto a Asia (que ocupa como región el tercer lugar), destacan países como China, Japón y Corea del Sur. En el caso de África, Medio Oriente y Países del Golfo, el país más visible es Israel.

Con estos datos podemos concluir que entre los años 2011 y 2015 (cuando el país se encontraba a la espera de la sentencia de la Corte Internacional de Justicia en La Haya sobre el diferendo marítimo con Chile) Perú.21 ha definido la política exterior correspondiente a la gestión de dicho período (Presidencia y Cancillería) como un intento de impulsar la integración (tanto política como comercial) en América del Sur, sobre todo con países 
como Venezuela, Bolivia, Ecuador y Brasil (2011-2013); mientras que, en cuanto a la Alianza del Pacífico (creada a iniciativa del ex presidente Alan García) lo ha buscado hacer en materia comercial con Chile, Colombia y México. Asimismo, se buscó consolidar las relaciones con Alemania, Rusia, China y Corea del Sur (2014-2015).

Aparecen países que, pese a no figurar en la lista de Cancillería, no han representado impedimento para que la gestión de ese entonces mantuviese conversaciones diplomáticas (Líbano y Palestina). Finalmente, otros dos elementos importantes que se presentan como los principales problemas de Perú21: si bien la mayor parte de los textos obedece a un trabajo de reporterismo (y no de "volteo" de notas de prensa o comunicados oficiales) predomina la utilización de un género tan básico como la noticia, fundamentalmente de tamaño breve (a comparación del reportaje a profundidad en más de una página) y la poca contextualización gráfica de sus textos.

\section{Fuentes consultadas}

Amadeo, B. (2008). La teoría del framing. "Los medios de comunicación y la transmisión de significados". Revista de Comunicación, núm. 1, pp. 6-32.

Armentia, J. y Caminos J. (1998). La información. Redacción y estructuras. Bilbao: Universidad del País Vasco.

Aruguete, N. y Zunino, E. (2010). "El encuadre de las noticias". En Luchessi, L. (comp.). Nuevos escenarios detrás de las noticias. Agendas, tecnologías y consumos. Buenos Aires: La Crujía.

Bajoit, G. (2003). Todo cambia. Análisis sociológico del cambio social y cultural en las sociedades contemporáneas. Santiago de Chile: LOM ediciones.

Bobbio, N.; Matteucci, I. y Pasquino, G. (1997). Diccionario de política. México D. F.: Siglo XXI Editores.

Bunge, M. (2007). Diccionario de filosofía. México D. F.: Siglo Veintiuno Editores.

D’Adamo, O. y García Beaudoux, V. (2009). "Medios y ciudadanos: percepción pública del comportamiento de los medios de comunicación de masas durante la campaña para las elecciones presidenciales de 2007 en Argentina". PostData, núm. 14, pp. 65-95.

DeVreese, C. (2005). "News framing: Theory and Tipology”. Information Design Journal, núm. 13, pp. 51-62.

Entman, R. (1993). "Framing: Toward a Clarification of a Fractured Paradigm". Journal of Communication. Vol. 43, núm. 3, pp. 51-58. 
Gamson, W. y Modigliani, A. (1989). "Media Discourse and Public Opinión on Nuclear Power: a Constructionist Approach”. American Journal of Sociology, núm. 95, pp. 1-37.

García Marín, M. (2007). El comportamiento de la prensa durante los conflictos de Kosovo e Irak [tesis doctoral]. Granada: Universidad de Granada. Extraída el 18/VIII/2016 desde http://hera.ugr.es/tesisugr/17098658.pdf?origin=publication_detail

Gomis, L. (2008). Teoría de los géneros periodísticos. Barcelona: UOCpress.

\section{Gonzales García, C.}

_(2016). Encuadres noticiosos en la información periodística sobre las relaciones internacionales del Perú según el diario Perú. 21 (julio 2011-julio 2015) [tesis de maestría] Lima: USMP, Facultad de Ciencias de la Comunicación, Turismo y Psicología.

_(2015). Tratamiento periodístico de las relaciones internacionales del Perú desde la perspectiva del framing [tesis de maestría]. Lima: UNMSM, Facultad de Letras y Ciencias Humanas.

Igartua, J. y otros (2005). "La información sobre inmigración e inmigrantes en la prensa española. Una aproximación empírica desde la teoría del encuadre”, pp. 75-85. En Fidalgo, A. y Serra, P. (eds.). Ciênccas da comunicaçâo en congresso na Covilhã. Teorias e estrategias discursivas. Covilhã: Universidade da Beira Interior.

Muñiz, C. (2015). "La política como debate temático o estratégico. Framing de la campaña electoral mexicana de 2012 en la prensa digital mexicana”. Nueva época, núm. 23, pp. 67-95.

Murciano, M. (1992). Estructura y dinámica de la comunicación internacional. Barcelona: Bosch.

Ochoa, O. (2000). Comunicación política y opinión pública. México D. F.: McGraw-Hill Interamericana.

Pearson, F. y Rochester, J. (2000). Relaciones Internacionales. Situación global en el siglo XXI. México D. F.: McGraw-Hill Interamericana.

Peralta, D. y Urtasun, M. (2007). La crónica periodística. Lectura crítica y redacción. Buenos Aires: La Crujía.

Pinto, M. (2009). “Análisis de la construcción mediática sobre la juventud en España. El tratamiento en las noticias en prensa, radio y televisión durante el 2006". Mediaciones Sociales, núm. 4, pp. 247-275.

Prieto L. (2013). "Tratamiento informativo del inicio de la crisis económica mundial de 2008 según la prensa nacional”. Extraída el 18/VIII/2016 desde http://www.razonypalabra.org.mx/N/N84/V84/25_Prieto_V84.pdf 
Rodrigo Alsina, M. (2005). La construcción de la noticia. Barcelona: Paidós.

Rodríguez Pérez, C. (2015). “La teoría del framing aplicada al 'rescate' a España”. Marco. Márketing y Comunicación Política, núm. 1, pp. 63-81.

Sádaba, T. (2001). “Origen, aplicación y límites de la 'teoría del encuadre’ (framing) en comunicación”. Comunicación y sociedad. Vol. 14, núm. 2, pp. 143-175.

Sánchez-Ancochea, D. (2007). "La globalización en países pequeños en desarrollo: Nicaragua en perspectiva comparada". Revista Mexicana de Sociología, núm. 2, pp. 199242.

Smelser, N. (2003). "Presiones para la continuidad en el contexto de la globalización". Revista Colombiana de Sociología, núm. 21, pp. 103-115.

Tankard, J. (2001). "The empirical approach to the study of media framing”, pp. 95-106. En Reese, S.; Gandy, O. y Grant, A. (eds.). Framing Public Life: Perspective on Media and our Understanding of the Social World. Nueva Jersey: Lawrance Erlbaum.

Urreiztieta, M. (2004). "La sociología interpretativa: globalización y vida cotidiana". Espacio Abierto. Vol. 13, núm. 3, pp. 457-490. Extraída el 18/VIII/2016 desde http://www.redalyc.org/articulo.oa?id=12213306

Verón, E. y otros (1971). Lenguaje y comunicación social. Buenos Aires: Nueva Visión.

Vicente, M. y López, P. (2009). "Resultados actuales de la investigación sobre framing: sólido avance internacional y arranque de la especialidad en España”. Zer, núm. 26, pp. 13-34. Extraída el 23/VIII/2016 desde http://www.ehu.eus/zer/hemeroteca/pdfs/zer26-01vicente.pdf 